\title{
Die Interform im Poly-Werk
}

Durch die teils anspruchsvollen und formal mehrbödigen Organisationsstrukturen kommt der Form im Poly-Werk auf dreifache Weise der Status einer grundlegenden Kategorie zu: erstens als Metaform, die die Konzeption des Ganzen umfasst; zweitens als Interform, die die Verhältnisse zwischen den Werken und ihre Situierung im Ganzen bestimmt, und drittens als Intraform, womit hier die konventionelle lineare Formrichtung des einzelnen Werks gemeint ist.

Im Mittelpunkt des folgenden Kapitels stehen die Interform, die die Möglichkeiten der simultanen Werkverbindung fokussiert, die Konvergenz und Divergenz der Werke, ihre gegenseitige Ergänzung als entweder stützende oder schwächende Kraft und die hieraus entstehenden Synergieeffekte. Die Möglichkeiten der simultanen Werkverbindung spiegeln einerseits die Spannbreite an Beziehungsmöglichkeiten des Zu-, Mit-, Neben- oder Gegeneinanders wider, situieren aber ebenso das einzelne Werk im Ganzen, seine Integration in den Werkzusammenhang, die mit unterschiedlichen Funktionen - beispielsweise der eines Solos oder eines Ensembleparts - einhergeht.

Um innerhalb eines simultanen Werkkontextes voneinander unabhängige Werke hervorzubringen, geht vielen Poly-Werken ein Prozess präkompositorischer Planung voraus. Manche Poly-Werke wachsen gar in einem jahrelangen Entstehungszeitraum. Auf den ersten Blick handelt es sich um ein sehr diverses Feld, das von sehr unterschiedlichen Komponisten und Komponistinnen individuell ausgelotet wurde. Mahnkopfs Zuschreibung eines höchst anpassungsfähigen und offenen Formkonzepts ${ }^{1}$ charakterisiert bereits die existierende Vielförmigkeit. Dennoch stehen die meisten Komponisten vor demselben Spagat, gleichzeitig das Werk als Einzahl und das Werk als Vielzahl zu realisieren.

Viele Komponisten haben ihre Ideen in räumlichen Bildern und Modellen, die außermusikalischen Terrains entlehnt sind und die simultane Disposition der Stücke zueinander in der Vorstellung abbilden, ausgedrückt. Luciano Berio vergleicht den Zyklus der Chemins, der den Zyklus der Sequenzae in sich aufnimmt, mit den Schichten einer Zwiebel: „[...] distinctes, séparées et pourtant collées l'une sur l'autre, chaque couche nouvelle créant une surface nouvelle, bien que rattachée à la précédente, et chaque couche ancienne assumant

1 Vgl. Mahnkopf: Kritik der neuen Musik, S. 118.

(C) KARIN WETZEL, 2022 | DOI:10.30965/9783846766743_004

This is an open access chapter distributed under the terms of the CC BY-NC-ND 4.o licensen wetzel - 9783846766743 
une nouvelle fonction dès qu'elle est recouverte .....2 Für Robert HP Platz sind naturwissenschaftliche Vorstellungen prägend: „Mich hat der Gedanke fasziniert, dass ,Up, Down, Strange and Charm‘ physikalische Teilchen, so genannte Quarks, sind, die je nach ihrem Anregungszustand entweder separat oder im Verbund auftreten können. Und das entspricht ganz genau der Aufführungsmöglichkeit meiner Stücke."3 Für Claus-Steffen Mahnkopf ist das Poly-Werk eine Spiegelung des „heutige[n], plurale[n] 'Mensch[en] [...], der in unvereinbare Mehrfachexistenzen aufgespalten zu sein scheint und dennoch ,irgendwie‘ ein Eines ist“4. Aber auch das Verhältnis verschiedener Individuen zueinander findet im Formkonzept des Poly-Werks seinen Ausdruck. Der Titel des Ensemblestücks „... Einklang freier Wesen ...“ von Georg Friedrich Haas entstammt einem Text von Hölderlin. Das Stück besteht aus zehn völlig in sich selbstständig aufführbaren Instrumentalstimmen, die dann unter dem Titel „... aus freier Lust ... verbunden ...“ zu beliebigen Ensembleformationen verbunden werden können. Haas knüpft nach Reinhard Kager an das Modell eines Zusammenschlusses „mündiger, selbstständiger Individuen [an], die nicht unters Joch eines Systems gespannt wären, ob dies nun Kommunismus heißt oder Kapitalismus“ ${ }^{5}$.

All diesen Vorstellungen und Stücken ist gemeinsam, dass sie eine neue formale Ebene benötigen, die auf die Verbindung und Verknüpfung der Einzelwerke gerichtet ist und die im folgenden Kapitel thematisiert wird. Ich nenne

2 Vgl. Luciano Berio, Paris, 19.6.1979, zitiert nach Ivanka Stoianova: Luciano Berio - Chemins en musique (= La Revue Musicale 375-377), Paris: Richard-Masse, 1985, S. 431.

3 Egbert Hiller: „Formpolyphonie als Zukunftsmusik. Der Komponist Robert HP Platz“, in: Neue Zeitschrift für Musik 6, 2005, S. 54-55, hier: S. 54.

4 Mahnkopf: Kritik der neuen Musik, S. 118. Dieser Satz findet sich bezogen auf das Lebenskunstwerk (LKW) fast identisch auch bei Paolo Bianchi und wurde im selben Jahr im Kunstforum International veröffentlicht: „Das LKW ist ein Flickwerk, das die Vielfalt und den Irrsinn des heutigen pluralen Menschen (und nicht eindimensionalen Menschen) spiegelt, der aufgespalten ist in unvereinbare Mehrfachexistenzen und dennoch irgendwie eine Einheit bildet." (S. 8) Bianchi verwendet ebenfalls den Begriff des Polywerks, allerdings bezogen auf das viel freiere und offenere Lebensgesamtkunstwerk (LKW) und nicht auf simultane Werkkonstellationen in der Musik: „Das LKW ist ein Polywerk, ein Werk, das sich aus mehreren Werken zusammensetzt, ein Werk, das andere Werke integriert. Keinerlei Sieg und keine Eindeutigkeit ist gefragt, sondern die Unendlichkeit von Beziehungen. Die Integration diverser Werke führt zur Simultaneität (statt zur Simulation) und erhöht die Interaktivität." (Paolo Bianchi: „Das LKW. Vom Gesamtkunstwerk zum Lebenskunstwerk oder ästhetisches Leben als Selbstversuch [Teil 1]“, in: Kunstforum 142, 1998, S. 50-61, III. „Crossover oder Hybrides, Simultanes und Polymorphes“, https://www.kunstforum.de/artikel/das-lkw/, S. 7 f. [letzter Aufruf am 7.5.2020].)

5 Georg-Friedrich Haas: „... Einklang freier Wesen ... “, Klangforum Wien, Sylvain Cambreling, Kairos, 2002, CD-Beiheft, S. 4. 
diese Ebene eine interformale Ebene. Sie kann wie ein Querschnitt profilhaft bestimmt werden und ermöglicht eine Gruppierung, Vergleichbarkeit und Einordnung der mitunter sehr unterschiedlichen Strategien. Die hieraus entstehenden, teilweise binären Reduktionen machen übergreifende Tendenzen und Merkmale sichtbar und systematisieren die vertikalen Werkverhältnisse. Dadurch entsteht ein relativ klarer Überblick über die Möglichkeiten. Dennoch gibt es auch Beispiele, die sich gerade nicht so einfach einordnen lassen. Manche Werke sind interessante Mischformen, auf die teilweise auch hingewiesen wird. Andere Werke wiederum schaffen Paradoxien. Eine eindeutige Zuordnung, wie sie Modelle gerne suggerieren, ist nicht in jedem Fall sinnvoll. Trotzdem schafft die Systematisierung die Voraussetzung, mit diesen Kategorien bewusster umzugehen, neue Mischformen zu erdenken und interessante Zwischenräume zu erkennen. Das Schema ist offen für Erweiterungen und soll in erster Linie als Leitfaden dienen, um ein PolyWerk in seinen formalen Grundzügen einordnen zu können. Es umfasst die Ebenen des Materials, des Werks, der Form und der Polylinearität. Das interformale Profil ersetzt keine vollständige Formanalyse. Diese kann erst aus dem Zusammenspiel der intra-, inter- und metaformalen Verläufe, Eigenschaften und Wechselwirkungen erwachsen.

\subsection{Homogene und heterogene Werkzyklen}

Es gibt zwei Gruppen von Poly-Werken, die sich aufgrund ihrer Materialverwandtschaft in homogene und heterogene Werkzyklen gruppieren lassen.

1. Homogene Werkzyklen

Bei der Zusammenführung homogener Werke stammen die einzelnen Werke von einem einzigen oder einem sehr ähnlichen Ausgangsmaterial ab oder basieren auf einer einzigen „Urform“.

Letzteres ist der Fall, wenn dem Zyklus ein verbindendes Ausgangsstück oder -modell zugrunde liegt und die Folgewerke als Ableitungen, Bearbeitungen, Transkriptionen oder Variationen dieser "Urform“ konzipiert sind. Ein Beispiel dafür ist der Yuunohui Cycle von Julio Estrada (vgl. Kapitel 4.2.1.2.1). Diesem Zyklus liegt als Modell eine grafische Struktur zugrunde, die auf die verschiedenen Versionen gleichermaßen übertragen wird, das heißt die entstehenden Versionen sind auf eine einzige Quelle zurückführbar und Variationen desselben Modells. Sie können entweder einzeln gespielt oder in beliebiger Anzahl simultan miteinander kombiniert werden. Auch Simon Steen-Andersens Zyklus Next To Beside Besides (vgl. Kapitel 4.2.1.2.2) und Juliane Kleins 
Werkserie Aus der Wand die Rinne (vgl. Kapitel 4.2.1.1.2) verfolgen ähnliche Ansätze. Beiden Werkclustern liegt ein Cellostück zugrunde, das als Initialstück am Anfang der Serie steht. Die anschließend komponierten Stücke für andere Soloinstrumente sind zu unterschiedlichen Graden Ableitungen und Variationen des Cellostücks. Sämtliche Stücke können sowohl einzeln als auch simultan gespielt werden. Gerade bei diesen sukzessive entstehenden Werkzyklen mit einer gemeinsamen Wurzel, die als Work in progress innerhalb eines mehrjährigen Entstehungszeitraums komponiert werden, kann es interessant sein zu sehen, inwieweit Differenzen zwischen den Stücken entwickelt werden und sich die Stücke im Verlauf des Entstehungszeitraumes auch voneinander "wegentwickeln" können. Typisch hierbei ist, dass diese Herangehensweise keine im Vorfeld gesetzte Metaform voraussetzt. Diese bildet sich immer wieder neu und kann daher als resultierende Form bezeichnet werden. ${ }^{6}$ Die beiden Stücke des Werkpaars Hängebrücken für zwei Streichquartette von Adriana Hölszky (vgl. Kapitel 4.2.2), die sowohl einzeln als auch simultan gespielt werden können, sind auf eine andere Art sehr eng verwandt. Beide Stücke verstärken sich und intensivieren einander. Im Gegensatz zu den oben beschriebenen Werkclustern gibt es hier kein eigentliches „Urmodell“. Dennoch schöpfen beide Werkstränge aus einem gemeinsamen Materialfundus, der im Skizzenstadium entwickelt und dann von Hölszky auf beide Werkstränge verteilt wurde.

2. Heterogene Werkzyklen

Bei der Zusammenführung heterogener Werke basieren die Teilwerke auf unterschiedlichem Ausgangsmaterial und sind daher in ihrer Werkidentität sehr verschiedenartig.

Beispielsweise führt Klaus Huber in seinem Orchesterstück Protuberanzen die drei kleinen Orchesterminiaturen Die Enge des Marktes, Implosion und Stäubchen aus Licht zusammen, die in ihrem Material und ihrem Charakter von vornherein unterschiedlichen Ideen entstammen und nicht die Vermehrfachung einer einzigen Idee verkörpern (vgl. Kapitel 4.3.4). Auch die Streichquartette Nr. 14 \& 15 von Darius Milhaud, die sich zum Oktett zusammenschließen lassen (vgl. Kapitel 4.3.1), exponieren unterschiedliche und kontrastierende Charaktere, die teilweise sehr unvermittelt aufeinanderprallen. Weitere Beispiele für heterogene Werkzusammenführungen sind der Anea Crystal Cycle von Chaya Czernowin

6 Eine Ausnahme bildet der Zyklus Next To Beside Besides von Simon Steen-Andersen. Denn hier wird die einmal gesetzte Form für alle Versionen beibehalten. 
(vgl. Kapitel 4.3.2), der Medusa-Zyklus von Claus-Steffen Mahnkopf (vgl. Kapitel 4.1.2) oder die Echoräume von Ulrich Kreppein (vgl. Kapitel 4.3.5). Um die heterogenen Werkverläufe zu einem Werk zusammenführen zu können, ist für diese Gruppe eine sehr plastische, schlüssige und architektonische Metaformbildung - sowohl bei den simultanen Fassungen als auch bei den Einzelfassungen - typisch. Anders als bei den homogenen Poly-Werken ist hier die Bildung von Brücken und Verbindungen zwischen den Stücken in der Analyse interessant.

\subsection{Endliche und nichtendliche Werkzyklen}

Die Unterscheidung von endlichen und nichtendlichen Werkzyklen leitet sich von der Frage ab, ob ein Poly-Werk-Zyklus als Work in progress theoretisch ins Unendliche wachsen kann oder ob von vornherein ein Rahmen gesetzt wird: entweder durch eine Maximalbesetzung, die nicht überschritten werden kann, oder durch eine vorab festgelegte Metaform, die die verschiedenen Werkverläufe festlegt und damit den zu vergebenden „Raum“ genau aufteilt.

1. Nichtendliche Werkreihen

Gerade bei homogenen Werkreihen, die auf Transkriptions- und Vervielfältigungsprozessen eines Ausgangsstückes basieren, ist es typisch, dass die Reihen in einem mehrjährigen Prozess nach und nach entstehen und in ihrer Anlage theoretisch unendlich erweiterbar und eigentlich nie abgeschlossen sind. Der bereits beschriebene Yuunohui Cycle von Julio Estrada (vgl. Kapitel 4.2.1.2.1) ist ein Beispiel für einen „unendlichen" wachsenden Kompositionszyklus. Der Entstehungszeitraum des Zyklus erstreckt sich mittlerweile über einen Zeitraum von fast vierzig Jahren. 1982 entstand das Cellostück Yuunohui'yei als Nukleus der Reihe. Weitere Fassungen für Violine, Viola, Kontrabass, Noisemaker, Cembalo, Orgel folgten in den 8oer Jahren. 2012 entstand mit Yuunohuiehecatl eine Version für Blasinstrumente, 2018 mit Yuunohui'sa eine Version für Gesang. Die ursprünglich sehr strenge Struktur der Streicherfassungen wurde dabei nach und nach zugunsten einer sehr viel offeneren Struktur, die den Performern mehr Freiräume und Optionen lässt, aufgegeben.

2. Endliche Werkreihen

Diesem Ansatz entgegengesetzt ist das „endliche Werk“, das in der Regel eine vorab bestimmte Rahmenbesetzung nicht überschreitet. Ein Gegenbeispiel zum Yuunohui Cycle stellt das Ensemblewerk „,... Einklang freier Wesen ..." (vgl. Kapitel 4.2.1.1.3) von Georg Friedrich Haas dar. Das Werk setzt sich aus zehn Solostimmen zusammen. Diese können einzeln 
aufgeführt werden oder sich zu beliebigen simultanen Kombinationen innerhalb dieser Rahmenbesetzung frei zusammenschließen. Statt eines Wachstumsprozesses erscheint dieses Werk als ein geschlossener Organismus, der sich ins Innere werkhaft ausdifferenziert, wobei die Zahl der Kombinationsmöglichkeiten durch den eindeutig definierten Gesamtrahmen klar begrenzt ist.

Ob ein Zyklus nichtendlich oder endlich ist, muss nicht in jedem Fall eindeutig zu bestimmen sein, denn einerseits verfügt der Komponist ja theoretisch immer über die Möglichkeit, im Nachgang Werke hinzuzufügen, andererseits ist auch sein Leben endlich, sodass eine „unendliche Fortsetzung“ eines Zyklus natürlich utopisch ist. Die Unterscheidung ist also eher auf eine mögliche Intention des Komponisten gerichtet: Entweder stellt sich der Werkzyklus aus Sicht des Komponisten als unabschließbar dar und es existiert die offene Option, weitere Versionen und Werke hinzuzufügen, oder das Werk stellt sich aus seiner Sicht an einem bestimmten Punkt als abgeschlossen in dem Sinne dar, dass die Hinzufügung weiterer Stücke dem Zyklus keinen Mehrwert mehr geben würde.

\subsection{Monozentrische und multizentrische Werkzyklen}

Hier ist zu bestimmen, ob die einzelnen Werke in einem hierarchischen oder eher einem gleichrangigen Verhältnis zueinander stehen. Gibt es beispielsweise ein zentrales Hauptwerk, an das kleinere Nebenwerke andocken können, dann handelt es sich um eine monozentrische Konstellation. Verbinden sich mehrere gleichrangige Werke, die einander auch in ihrer Besetzungsstärke und Dauer ungefähr entsprechen, dann handelt es sich um eine multizentrische Konstellation, da kein Werk dem anderen übergeordnet ist. Statt monozentrisch/multizentrisch könnte hier je nach Werk auch die Unterscheidung Parataxe versus Hypotaxe sinnvoll sein. Mit Parataxe ist ein ebenbürtiges Verhältnis "gleichrangiger" Stücke gemeint, während Hypotaxe auf eine integrativ-verschachtelte Form zielt, die beispielsweise kleine Nebenwerke in ein größeres Hauptwerk integriert.

1. Multizentrische Werkzyklen

Die multizentrale Konstellation ist „nichthierarchisch“. Hier handelt es sich um eine Verbindung von mindestens zwei Stücken, die in einem gleichrangigen Verhältnis zueinander stehen. Gleichrangig bedeutet erst einmal kein Werturteil über die Stücke an sich. Gemeint ist, dass die Werke in ihrer Besetzung, ihrer Dauer und ihrem zeitlichen Zusammenfall einander entsprechen, also gemeinsame äußere Merkmale haben 
und keine Werkversion einer anderen im Zusammenspiel übergeordnet erscheint, das Verhältnis ausbalanciert ist. Der Yuunohui Cycle von Julio Estrada geht von verschiedenen gleich starken Solostücken aus, die sich dann untereinander frei verbinden können. Sowohl jedes Solostück als auch jede zusammengesetzte Version hat ihren individuellen Charme und lässt jede Stimme gleichberechtigt zu Wort kommen. Keine Version scheint einer anderen übergeordnet zu sein, wirkt stärker oder schwächer, sodass sich hier von einer tatsächlichen Gleichrangigkeit sprechen lässt.

2. Monozentrische Werkzyklen

Eine monozentrische Werkdisposition geht meistens mit einer Hierarchiebildung unter den Werken einher. Innerhalb einer Werkentität können die Werke beispielsweise in Haupt- und Nebenwerke unterteilt werden. Claus-Steffen Mahnkopf verwendet für die Kammermusikstücke seines Orchesterwerks Medusa etwa den Begriff des "Subwerks" ${ }^{4}$. Darin schwingt bereits eine untergeordnete Stellung der Werke im Gesamtkontext mit. Zwar handelt es sich bei den Solo- und Kammermusikstücken nicht um „schwache“ Versionen - in dem Moment, in dem sie für sich stehen und sich der Wahrnehmungsfokus auf sie richten kann, sind sie in sich stimmig -, in einer Gesamtaufführung werden sie vom Orchester aber teilweise einfach geschluckt, insbesondere das Cembalostück Pegasos geht in der Orchesterversion gnadenlos unter.

Auch ob es sich um eine mono- oder multizentrische Werkdisposition handelt, ist nicht bei jedem Poly-Werk eindeutig und objektiv zu bestimmen. Denn es geht ja nicht nur um die Frage, welche Werke sich aufgrund ihrer Besetzungsstärke oder Satzdichte akustisch am besten durchsetzen können, sondern auch darum, inwieweit es sich um qualitativ stärkere oder schwächere Versionen handelt. Diese Frage schneidet das Feld der Interpretation und der Rezeption, unterliegt also auch einer subjektiven Bewertung. Beispielsweise handelt es sich bei dem Zyklus „... Einklang freier Wesen ..." von Georg Friedrich Haas um eine multizentrische Anlage, da sich zehn für sich stehende Einzelstimmen auch untereinander zu dem Stück mit dem Titel „... aus freier Lust ... verbunden ..." zusammenschließen können. Dennoch würde ich dazu tendieren, die Gesamtfassung ,... Einklang freier Wesen ... “, die alle zehn Stimmen umfasst, in der Rezeption als eine Hauptfassung wahrzunehmen, dagegen die Aufführung einer Einzelstimme als eine Nebenfassung. Damit würde dieses Werk beide Möglichkeiten in sich tragen. Mit der Gesamtfassung hätte das Werk ein eindeutiges Zentrum. Die anderen Versionen haben - im Vergleich zur

7 Mahnkopf: „Medusa“, S. 245 . 
Hauptfassung - zwar eine Nebenstellung, sind untereinander aber mehr oder weniger gleichrangig und damit multizentral.

\subsection{Möglichkeiten der zeitlich-horizontalen Lageposition}

Dieser Punkt betrifft das Verhältnis von Sukzessivität und Simultaneität, meint also das Ausmaß der zeitlich horizontalen Ineinanderschiebung der Werke. Die Spannbreite reicht von einer Überlappung, die nur wenige Takte umfasst, bis zu einer vollständigen Überlappung. Eine nur wenige Takte andauernde Überlappung ist meines Erachtens zu marginal, um unter der Rubrik „PolyWerk" thematisiert zu werden. Kompositorisch stellt sie nicht die gleiche substanzielle Herausforderung bezüglich der Verknüpfung von Werken dar wie das Übereinanderlegen längerer Abschnitte. Die folgenden drei Möglichkeiten umfassen erstens die teilweise Überlappung - wobei als Faustregel gilt, dass die simultane Dauer gegenüber der Einzel- oder der rein sukzessiven Dauer als dominierend wahrgenommen wird -, zweitens die verschachtelte Überlappung - die die Möglichkeit vorsieht, die Einzelstücke in zeitliche Abschnitte zu schneiden und diese in ihrer Originalreihenfolge über die Gesamtdauer zu verteilen - und drittens die vollständige Überlappung.

1. Teilüberlappungen

Die Werke überlappen sich nur für einen Teil ihrer Dauer. Diese simultane „Teilstrecke“ sollte nicht viel kürzer sein als die „Einzelstrecke“, damit die Simultaneität auch tatsächlich einen Effekt entfalten kann. Ein Beispiel für eine simultane Teilüberlappung stammt von Klaus Huber, dessen Protuberanzen drei Orchesterstücke vereinen, die sowohl sukzessive als auch simultan überlappend aufführbar sind (vgl. Kapitel 4.3.4). Dieser Zusammenführung liegt eine genau kalkulierte Konzeption zugrunde, die die Voraussetzung für die Synthese der drei Stücke zu einem Stück ist.

2. Verschachtelung

Die zweite Möglichkeit umfasst Stücke, die zwar vollständig Teil einer Simultaneisierung sind, aber in der simultanen Form nicht am Stück durchgespielt werden. Stattdessen werden sie in Abschnitte geteilt, die über die Gesamtdauer verteilt werden. Dadurch ergibt sich eine formale Ineinanderverschachtelung der Stücke, die auch Pausen und Unterbrechungen in den musikalischen Text einlässt, die ein reizvolles dramaturgisches Gestaltungsmittel sein können. Als Beispiel kommen die Echoräume von Ulrich Kreppein in Betracht (vgl. Kapitel 4.3.5). Hier vereinigen sich vier Kammermusikstücke zu einem Ensemblestück. Die Stücke werden nicht durchgehend gespielt, sondern in kurze Abschnitte 
zerteilt, die der Gesamtdramaturgie entsprechend über die Gesamtdauer von zwanzig Minuten gelegt werden. Mithilfe eines Planes und der Angabe von Orientierungszeiten werden die jeweiligen Einsatzpunkte für die vier Ensembles vermittelt.

3. Vollständige Überlappung

Die Form einer vollständigen Überlappung ist die verbreitetste Variante. Anfangs- und Endpunkt der Stücke fallen zusammen, und auch im Verlauf gibt es keine Schnitte und Unterbrechungen. Beispiele sind die beiden Streichquartette Nr. 14 \& 15 von Darius Milhaud, der Zyklus „... Einklang freier Wesen ..." von Georg Friedrich Haas, der Yuunohui Cycle von Julio Estrada oder auch Simon Steen-Andersens Zyklus Next To Beside Besides.

\subsection{Möglichkeiten der vertikalen Lageposition}

Neben den verschiedenen Möglichkeiten der Lagepositionierung in der horizontal-zeitlichen Dimension gibt es auch verschiedene Optionen, die Stücke in ihrer Körperlichkeit zusammenzubringen, sie einander entweder frontal gegenüberzustellen oder sie miteinander zu verschränken. Grundsätzlich handelt es sich bei den meisten Vorgehensweisen um additive Praktiken. Aber auch die Subtraktion als umgekehrte Form der Addition ist möglich, wenngleich sie nicht immer eindeutig als solche zu bestimmen ist.

1. Einfache Addition

Bei der einfachen Addition handelt es sich um eine Gegenüberstellung der verschiedenen, oft "gleichrangigen“ Werke. Denn häufig entsprechen die Werke einander in ihrer Dauer und ihrer Besetzung und überlappen sich vollständig oder fast vollständig. Ähnlich wie die vollständige Überlappung ist die einfache Addition die verbreitetste Variante. Beispiele sind auch hier die beiden Streichquartette Nr. 14 \& 15 von Darius Milhaud, der Yuunohui Cycle von Julio Estrada oder auch Simon Steen-Andersens Zyklus Next To Beside Besides.

2. Integration

Integration bedeutet, dass „kleinere“ Stücke in eine größere Werkentität integriert werden. Es handelt es sich also um eine Zusammenführung ungleich starker Werkteile. So ist beispielsweise Claus-Steffen Mahnkopfs Medusa-Zyklus organisiert. Das Oboenkonzert als Dachkomposition nimmt verschiedene kleinere Subwerke, die solistisch oder kammermusikalisch besetzt sind, in sich auf. Die Solo- und Kammermusikstücke sind in ihrer Besetzung dem Orchesterstück zahlenmäßig unterlegen, haben aber auch eine kürzere Dauer. Weitere Beispiele sind 
Berios Chemins - eine Ensemblereihe, die einige der Sequenzae weiterkomponiert - und Richard Barretts Opening of the Mouth. Alle drei Beispiele sind unidirektional, das heißt das größere Werk kann ohne das aufzunehmende Werk nicht selbstständig aufgeführt werden (vgl. Kapitel 4.1).

3. Kreuzende Addition

Bei der kreuzenden Addition wird der musikalische Text zwar im Original beibehalten, aber einzelne musikalische Ebenen können in der Simultanversion bidirektional ausgetauscht und dadurch sehr eng miteinander verschränkt werden. Dieses Verfahren setzt bei den Werken in der Regel den gleichen zeitlichen Rahmen und die dieselbe Anzahl von Instrumenten voraus. Ein Beispiel stammt von Valerio Sannicandro. Seine Tactile Songs I-II (2017-2018) umfassen zwei Quartettreihen, die auch simultan gespielt werden können. Die erste Reihe ist mit Bassflöte, Violoncello, Gitarre und Percussion besetzt, die zweite Reihe mit Bassflöte, Klarinette, Viola und Percussion. Beide Quartette können eigenständig oder auch simultan aufgeführt werden und darüber hinaus auch in Fassungen, in denen die Instrumentenschichten teilweise ausgetauscht werden, sodass also Bassflöte und Percussion aus dem ersten Zyklus gleichzeitig mit Klarinette und Viola aus dem zweiten Zyklus gespielt werden. Durch diese zusätzliche Option können trotz einer zahlenmäßig festgelegten Rahmenbesetzung zusätzliche Aufführungsformen erzeugt werden. Auch mein Stück X-Pieces ist ein Beispiel für eine kreuzende Addition. Hier werden die Sologanzheiten in eine Artikulations- und Fingerebene aufgespalten, die werk- und spielerübergreifend austauschbar sind. Beispielsweise kann sich die Artikulationsebene des einen Stückes mit der Fingerstimme des anderen Stückes als Solo/Duo auf einer Flöte, gespielt von zwei Spielern, verbinden (vgl. Kapitel 5.2).

4. Subtraktion

Hier handelt es sich um eine Umkehrung des Integrationsprozesses, also die Herausnahme kleinerer Stücke aus einem größeren Werkkontext. $\mathrm{Ob}$ eine Schicht hinzugefügt oder weggenommen wird, ist einerseits lediglich eine Frage der Perspektive - denn ein Poly-Werk kann von zwei Seiten gedacht und gehört werden - und kann andererseits das Resultat einer komplexen Werkgenese sein. Entscheidet der Komponist erst im Nachhinein oder in einem weit fortgeschrittenen Stadium des Kompositionsprozesses, eine oder mehrere Stimmen aus einem größeren Kontext als selbstständige Werke herauszueinzeln, dann wird das "Mehr“ an Werkversionen tatsächlich durch eine Reduktion, ein Wegnahme von Schichten erzeugt. Klaus Hubers Trio Schattenblätter (1975) könnte 
ein Beispiel für eine subtraktive Werkgenese darstellen. Im Mittelpunkt steht die Trioversion. Es sind jedoch sowohl alle Duokombinationen als auch der asketische und karge Solopart des Klaviers mit dem Titel Blätterlos unabhängig aufführbar. ${ }^{8}$ Die Frage, ob eine Addition oder eine Subtraktion vorliegt, ist nicht immer eindeutig zu beantworten. Ich würde beispielsweise bei Georg Friedrich Haas' Zyklus ,... Einklang freier Wesen ..." dazu tendieren, die Herauseinzelung der Solo- und Kammermusikversionen als einen subtraktiven Prozess zu bezeichnen. Ähnlich wie bei Hubers Schattenblätter steht die Gesamtfassung als eine Art Hauptwerk und Mittelpunkt über dem Ganzen. Beide Werke sind nicht in einem Wachstumsprozess entstanden, wie es bei Julio Estradas Yuunohui Cycle der Fall ist, sondern gehen von einer klaren Rahmensetzung aus, die nicht überschritten, sondern nur unterschritten werden kann.

\subsection{Synchrone und asynchrone Prozesse}

Eine weitere Schlüsselkategorie im interformalen Verhältnis ist der Grad der Synchronität oder Asynchronität zwischen den einzelnen Werken, unabhängig von der Materialverwandtschaft. Dieses Kategorie verteilt sich auf die verschiedenen musikalischen Ebenen eines Stückes wie die Metrik, das Tempo, die Harmonik, die Form usw. Man könnte diesen Punkt auch als Polysierung bezeichnen, da es darum geht, den Grad der Unabhängigkeit verschiedener Verläufe zu bestimmen. Es ist immer die Frage, welche Ebenen synchronisiert, möglicherweise sogar identisch sind und auf welchen Ebenen die Differenzen zwischen den Stücken geschaffen werden. Jedes Poly-Werk kombiniert und ordnet diese Möglichkeiten für sich neu. Im Schnitt gibt es aber in allen PolyWerken Ebenen, die stark übereinstimmen, und Ebenen, die divergierende Verläufe ermöglichen. Wahrscheinlich ist gerade diese Ausgewogenheit zwischen Konvergenz und Divergenz die Voraussetzung, um auf der einen Seite keine zu große Übereinstimmung und auf der anderen kein beliebiges Chaos zuzulassen, sondern die konvergenten und divergenten Prozesse sehr bewusst gestalten zu können.

8 Klaus Huber hat seine Intention zu Werkreduktionen in einem anderen Zusammenhang einmal folgendermaßen beschrieben: „Während einige Komponisten dazu neigen, früher komponierte Werke dergestalt zu rekomponieren, daß sie allen denkbaren Ansätzen zur Erweiterung und Komplexierung nachspüren (exemplarisch Pierre Boulez in ,Notations'), bevorzuge ich seit mehr als zwanzig Jahren den umgekehrten Weg. Ich suche nach möglichen Reduktionen." Siehe auch Klaus Huber in: Huber/Nyffeler (Hg.): Umgepflügte Zeit, S. 442 . 
1. Dauer

Bereits die Dauer der Stücke ist eine wichtige Kategorie bei ihrer Zusammenführung. Entweder sie haben denselben zeitlichen Rahmen und können sich dadurch auch vollständig überlappen, oder sie unterscheiden sich in ihrer zeitlichen Dauer sehr stark voneinander, sodass für die Zusammenführung ein eigener Plan nötig wird, der festlegt, wie sich die Stücke entweder überlappen oder ineinander verschachtelt werden.

2. Instrumentation, Klangfarben, Spielpraxis

Die Ebene der Instrumentation betrifft einerseits die Anzahl der Besetzungen, aber auch die Frage, inwieweit sich identische, ähnliche oder sehr unterschiedliche Besetzungsformen gegenüberstehen, die den Grad der Verschmelzung zwischen den Werken entweder fördern oder auf Kontrast zielen.

Beispielsweise handelt es sich bei den beiden Streichquartetten Nr. 14 \& 15 von Darius Milhaud um zwei völlig unterschiedliche Werkidentitäten, was das Material betrifft. Trotzdem wird durch die gleiche Besetzung von zwei mal vier Streichern auch eine starke Verschmelzung der beiden Werke gefördert (vgl. Kapitel 4.3.1). Ein Gegenbeispiel stammt von Chaya Czernowin. Ihr Anea Crystal Cycle ist ebenso für zwei Streichquartette komponiert, die auch gleichzeitig gespielt werden können. Czernowin allerdings unterstreicht die Heterogenität des Materials durch die unterschiedlichen Spieltechniken beider Quartette, zum Beispiel Pizzicatospiel im ersten und Arcospiel im zweiten Quartett. Dadurch sind beide Quartette bei einer Simultanaufführung über weite Strecken gut voneinander abgrenzbar und unterscheidbar (vgl. Kapitel 4.3.2).

3. Metrum

In der Regel ist das Metrum der Puls des Stückes und daher eine sehr zentrale Kategorie in der zeitlich-organisatorischen Gestaltung. Entweder die Werke sind metrisch synchronisiert oder polymetrisch. Die meisten Poly-Werke teilen dasselbe Metrum. In Chaya Czernowings Anea Crystal Cycle sind die Metren dagegen teilweise extrem gegensätzlich. Die Heterogenität des Materials wird so durch eine zeitliche Heterogenität unterstrichen. Während das zweite Quartett in geradtaktigen Metren gruppiert ist, ist die Metrik des ersten Quartettes sehr stark von Doppelbruchmetrik geprägt (vgl. Kapitel 4.3.2).

4. Tempo

Auch das Tempo ist ein Schlüsselfaktor auf der zeitlich-organisatorischen Ebene. In den meisten Poly-Werken sind die Teilwerke im Tempo synchronisiert. Nicht jedoch im Anea Crystal Cycle von Chaya Czernowin. 
Die Tempoangaben für beide Quartette sind unterschiedlich und wechseln zu unterschiedlichen Zeiten. Auch in den Echoräumen von Ulrich Kreppein sind die Tempi für jedes Einzelwerk individuell (vgl. Kapitel 4.3.5).

5. Lineare Formprozesse

Der Formverlauf der Einzelwerke, ihre Ereignistemporalität, ist als Polylinearität das wahrscheinlich aufschlussreichste Kriterium, um den Grad der Individualität der Einzelwerke zu bestimmen. Sind Zäsuren, Formabschnitte, Höhepunkte, dramaturgische Entwicklungen etc. über die Werkgrenzen hinweg synchronisiert oder gerade nicht? Ein wesentlicher Grund für die kohärente Wirkung des Werks „... Einklang freier Wesen ..." von Georg Friedrich Haas ist die Übereinstimmung der Abschnitte, Zäsuren und Entwicklungen, die für alle Instrumente verbindlich sind.

Für die Gestaltung der Formverläufe entfaltet der Begriff der Formpolyphonie, der mit den Werken von Robert HP Platz in Verbindung gebracht wird, hier seine eigentliche Bedeutung. Er bezeichnet entweder die Anwendung unterschiedlicher Formtypen innerhalb eines Ganzen oder den Entwurf individueller unterschiedlicher Formen, die in einem Poly-Werk miteinander kombiniert werden. In beiden Fällen zielt er auf eine Divergenz der kombinierten Formen. Im Medusa-Zyklus entwirft Mahnkopf entweder für jedes Einzelwerk eine individuelle Form oder wendet einen spezifischen Formtypus an, passt diesen aber präzise in die Gesamtform ein. Auch hier ist also der Fall einer Formpolyphonie gegeben. Das ist ebenso im Yuunohui Cycle von Julio Estrada der Fall. Simultane Formereignisse wie ein gemeinsamer Höhepunkt oder eine gemeinsame Zäsur entstehen hier vor allem zufällig zwischen den Soli. Estrada zielt trotz der übereinstimmenden Metrik, Tempi und Dauer eher auf ein zeitliches Nebeneinander in den linearen Formprozessen. Häufig liegen allerdings auch Mischformen vor, das heißt die Makrooder Metaform eines Werks ist beispielsweise für alle Teilwerke verbindlich, aber die mikroformalen Entwicklungen der einzelnen Abschnitte weichen voneinander $a b$ und sind individualisiert. Beispiele dafür sind die Streichquartette von Milhaud und der Anea Crystal Cycle von Czernowin.

6. Harmonik

Auch die harmonische Behandlung ist eine wesentliche Komponente in Bezug auf Individualität, Divergenz, Verschmelzungsfähigkeit etc. Georg Friedrich Haas hat für das Werk ,... Einklang freier Wesen ...“ im Vorfeld einen harmonischen Plan entworfen, an den alle 
Instrumentalschichten gebunden sind, das heißt alle Solostimmen folgen derselben Harmonik. Das lässt sie einerseits sehr gut miteinander verschmelzen, andererseits werden sie dadurch in ihrer melodischen Beweglichkeit sehr eingeschränkt (vgl. Kapitel 4.2.1.1.3).

Im Gegensatz dazu ist in Milhauds Streichquartetten der polytonale Tonsatz ein entscheidendes Kriterium, um Individualität und Divergenz zwischen den Quartetten zu erzeugen. Milhaud schichtet sorglos verschiedenste Tonarten übereinander und unterstreicht so die Heterogenität der Quartette. Ebenso ermöglicht ihm die Polytonalität, harmonische Brücken zwischen den Quartetten zu bauen. Durch die Kombination von Polytonalität und Poly-Werk wird harmonisch ein sehr hoher Grad an Mehrdeutigkeit erreicht (vgl. Kapitel 4.3.1). 